\title{
Gene, Environment and Methylation (GEM): a tool suite to efficiently navigate large scale epigenome wide association studies and integrate genotype and interaction between genotype and environment
}

\author{
Hong Pan ${ }^{1,2}$, Joanna D. Holbrook', Neerja Karnani ${ }^{1,3}$ and Chee Keong Kwoh ${ }^{2^{*}}$
}

\begin{abstract}
Background: The interplay among genetic, environment and epigenetic variation is not fully understood. Advances in high-throughput genotyping methods, high-density DNA methylation detection and well-characterized sample collections, enable epigenetic association studies at the genomic and population levels (EWAS). The field has extended to interrogate the interaction of environmental and genetic (GxE) influences on epigenetic variation. Also, the detection of methylation quantitative trait loci (methQTLs) and their association with health status has enhanced our knowledge of epigenetic mechanisms in disease trajectory. However analysis of this type of data brings computational challenges and there are few practical solutions to enable large scale studies in standard computational environments.

Results: GEM is a highly efficient $\mathrm{R}$ tool suite for performing epigenome wide association studies (EWAS). GEM provides three major functions named GEM_Emodel, GEM_Gmodel and GEM_GxEmodel to study the interplay of Gene, Environment and Methylation (GEM). Within GEM, the pre-existing "Matrix eQTL" package is utilized and extended to study methylation quantitative trait loci (methQTL) and the interaction of genotype and environment (GXE) to determine DNA methylation variation, using matrix based iterative correlation and memory-efficient data analysis. Benchmarking presented here on a publicly available dataset, demonstrated that GEM can facilitate reliable genome-wide methQTL and GXE analysis on a standard laptop computer within minutes.

Conclusions: The GEM package facilitates efficient EWAS study in large cohorts. It is written in R code and can be freely downloaded from Bioconductor at https://www.bioconductor.org/packages/GEM/.
\end{abstract}

Keywords: Matrix operation, EWAS, methQTL, GxE

\section{Background}

Understanding DNA methylation biomarkers of environmental exposures and developmental trajectories to disease is highly desirable [1] and their discovery is the aim of many epigenome wide association studies (EWAS) $[2,3]$. The computational burden in analyzing the genomics data from this type of studies is

\footnotetext{
* Correspondence: ASCKKWOH@ntu.edu.sg

${ }^{2}$ School of Computer Science and Engineering, Nanyang Technological

University (NTU), Singapore 639798, Singapore

Full list of author information is available at the end of the article
}

considerable due to the high number of variables returned from epigenetic screens, for instance $>483,000$ individual measures from the widely used Illumina Infinium HumanMethylation450 Array (Infinium450K) [4] or the millions of loci covered by RRBS [5] or methylcapture technologies [6, 7]. Hundreds or thousands of subjects are required to provide the statistical power to draw inference in EWAS studies [8]. The need to include covariates pertaining to the subjects, such as gender, ethnicity and social economic status [9], and to the samples, such as cellular heterogeneity [10-12], 
increase the computational time needed to run statistical models. Some of these problems are familiar from the genome wide association studies (GWAS) field, although DNA methylation profile is surrogated by continuous percentage values and distributed very differently from genotype calls.

However, what has really pushed EWAS studies to the brink of what is computationally possible, is the realization that DNA methylation levels are not just specified by extrinsic factors but also are influenced by genotype. Polymorphisms close to CpGs in the same chromosome (cis-) often form methylation quantitative trait loci (methQTLs) with nearby CpGs [13-15], or blocks of cis- polymorphisms associated with a cluster of methylation quantitative trait loci, named GeMES (15, 19). MethQTLs can be discovered by correlating single nucleotide polymorphism (SNP) data with CpG methylation from the same samples. Creating a genome wide methQTL map requires assessing the correlation of genotype at millions of SNPs with thousands to millions of CpG methylation states, by millions multiplied with millions linear iterative regressions. Sun 2014 [16] surveyed methQTL studies between year 2010-2014 and found that most of methQTL studies were restricted to screen cis- SNP-CpG pairs, while some were even restricted to the $50,000 \mathrm{bp}$ to $1,000,000 \mathrm{bp}$ regions flanking to each SNP. However SNPs far from the CpG or in different chromosome (trans-) were also reported to be associated with CpG. Trans- methQTLs have been detected to be relevant to normal or disease states in many studies [17].

Furthermore, it is now apparent that genotype can work in interaction with environment $(\mathrm{GxE})$ to influence specific DNA methylation levels $[18,19]$ and these can be linked to phenotypes [20,21]. This type of correlated methylation structure has implications for statistical models whereby genotype and environment, or genotype and methylation interact to predict methylation levels or phenotype. This has exponentially increased the computational burden for the proper analysis of EWAS data.

Large-scale genomic research benefits from highperformance computing (HPC) environments together with parallel computing techniques. However, the operation and integration of results needs domain expertise [22] and HPC is not always easily accessed by biology lab researchers. Therefore, we were motivated to develop computational solutions that allow biological researchers to explore EWAS, methQTLs and GxE using standard desktop computers within realistic computational times.

A R package called MatrixEQTL [23] was developed for expression quantitative train loci (eQTL) analysis. Based on matrix operation, iterative correlation was implemented to achieve computational efficiency, and data was sliced into blocks to achieve memory efficiency. A function in MatrixEQTL that allows inclusion of interaction terms in correlative statistical models, gained our attention, though the author did not highlight it when

Table 1 Pseudo R code for Emodel

Algorithm: exploring the association between methylation and environment factors by Emodel.

Input:

Methylation matrix $\boldsymbol{M}$ (number_of_CpGs $\times$ number_of_Samples), and each $M(i)$ is the vector for ith CpG cross all samples.

Environmental vector $\boldsymbol{E}$ (number_of_Samples), Covariate matrix curt (number_of_covariates $\times$ number_of_Samples).

Output:

A list of CpG-Env pairs

\begin{tabular}{lll}
\hline 1 & Function: LM_Emode $(\boldsymbol{M}, \boldsymbol{E}, \boldsymbol{c v r t})$ \\
2 & For i=1 to number_of_CpGs \{ \\
3 & & fit<-summary(Im(M(i) $\sim \boldsymbol{E}+\boldsymbol{c v r t}))$ \\
4 & \} & \\
\hline 5 & Function: GEM_Emodel $(\boldsymbol{M}, \boldsymbol{E}, \boldsymbol{c v r t})$ \\
6 & $\{\quad$ & library (GEM) \\
7 & & result $=$ GEM_Emodel $(\boldsymbol{M}, \boldsymbol{E}, \boldsymbol{c v r t})$ \\
8 & \} & \\
\hline
\end{tabular}


the package was reported. We deployed the fast and efficient MatrixEQTL software and created a tool suite to explore the associations of Gene, Environment and Methylation. We named the tool suite "GEM". It provides three fast linear regression models denoted Emodel, Gmodel and GxEmodel to facilitate analyses in EWAS. The GEM_Emodel tests the association of methylome marks and environmental factors; the GEM_Gmodel creates a methQTL genome-wide map; finally, the GEM_GxE model tests the ability of gene and environmental interaction models to predict DNA methylation levels. We benchmarked the performance of the GEM operations on a publicly available EWAS dataset generated on the Infinium450K array with concurrent genotyping on the OmniExpress Array and simulated environment and phenotype information on 237 neonates. Our results demonstrated that the GEM package can facilitate reliable EWAS analyses within minutes, in a

Table 2 Pseudo R script to explore methQTLs by Gmodel

Algorithm: exploring the methQTL by Gmodel.

Input:

Methylation matrix $\boldsymbol{M}$ (number_of_CpGs $\times$ number_of_Samples), and

each $\boldsymbol{M}(i)$ is the vector for ith $\mathrm{CpG}$ cross all samples.

Genotype matrix $\boldsymbol{G}$ (number_of_SNPs $\times$ number_of_Samples), and

each $\boldsymbol{G}(\mathbf{j})$ is the vector for $j$ th SNP cross all samples.

Covariate matrix curt (number_of_covariates $\times$ number_of_Samples).

Output:

A list of CpG-SNP pairs, where the SNP is the best fit to explain the particular CpG. The significant association between CpG-SNP pair suggests the methylation driven by genotyping variants, which is so called methylation quantitative trait loci (methQTL).

\begin{tabular}{|c|c|}
\hline 1 & Function LM_Gmode ( $G, M$, curt $)$ \\
\hline 2 & For i=1 to number_of_CpGs \{ \\
\hline 3 & For $\mathrm{j}=1$ to number_of_SNPs \{ \\
\hline 4 & fit<- summary $(\operatorname{lm}(M(i) \sim G(j)+$ cvrt $))$ \\
\hline 5 & \} \\
\hline & result $(i)=(M(i), G(K))$ by \\
\hline 6 & $\mathrm{~K}=\operatorname{argmax}_{k \in\left[1, \text { mumber_SNPs }_{3}\right]}($ fit $\$ \mathrm{r}$.squared) \\
\hline 7 & \} \\
\hline 8 & Function GEM_Gmode(G, $M$, curt $)$ \\
\hline 9 & $\{\quad \operatorname{library}(G E M)$ \\
\hline 10 & result = GEM_Gmodel $(G, M$, cvrt $)$ \\
\hline 11 & \} \\
\hline
\end{tabular}


standard computational setting (processor $=2.2 \mathrm{GHz}$, RAM $=8 \mathrm{G}$, system $=$ window7 64bit).

\section{GEM implementation}

Simplifying the data input into a methylation matrix as $\boldsymbol{M}$, genetic variants matrix as $\boldsymbol{G}$, and the environment vector as $\boldsymbol{E}$, and the matrix for covariates as curt, and using a pseudo coding language like R script, we can denote Emodel (detecting methylation markers associated with environment) function as $\boldsymbol{l m}(\boldsymbol{M} \sim \boldsymbol{E}+\boldsymbol{c v r t})$, Gmodel (detecting methylation markers associated with genotype i.e. methQTLs) as $\boldsymbol{l m}(\boldsymbol{M} \sim \boldsymbol{G}+\boldsymbol{c v r t})$ and $\mathrm{GxE}$ model (interaction of genotype and environment to specify methylation marks) as $\boldsymbol{l m}(\boldsymbol{M} \sim \boldsymbol{G} \times \boldsymbol{E}+\boldsymbol{c v r t})$. The genome wide studies for Emodel, Gmodel and GxEmodel can be accomplished by calling $\mathrm{R}$ function $\boldsymbol{l} \boldsymbol{m}$ iteratively by millions of times, which were denoted as LM_Emodel (Table 1), LM_Gmodel (Table 2) and LM_GxEmodel.

Shabalin [23] introduced matrix standardization and projection and successfully made an ultra-fast software for expression quantitative trait loci (eQTL). Basically, to quantify the strength of the relationship between $\boldsymbol{x}$ and $\boldsymbol{y}$ controlled by covariates (cvrt), a practical regression is,

$$
y=\alpha+\beta x+\gamma c v r t+\varepsilon
$$

where $\boldsymbol{\alpha}, \boldsymbol{\beta}, \boldsymbol{\gamma}$ and $\boldsymbol{\varepsilon}$ are coefficients, $\boldsymbol{\beta}$ is to estimated. A standardization method (22) was applied to vector $\boldsymbol{x}, \boldsymbol{y}$, $c v r t$, then the projections of $\boldsymbol{x}$ and $\boldsymbol{y}$ to $\boldsymbol{c} v \boldsymbol{r t}$ are,

$$
\begin{aligned}
& \tilde{\boldsymbol{y}}=\boldsymbol{y}-<y, \quad \text { cvrt }>\quad \text { cvrt, and } \\
& \boldsymbol{x}=\boldsymbol{x}-<x, \quad \text { cvrt }>\quad \text { cvrt },
\end{aligned}
$$

where $<>$ denotes inner product of two matrix. After these operations, the linear regression between $x$ and $y$ with

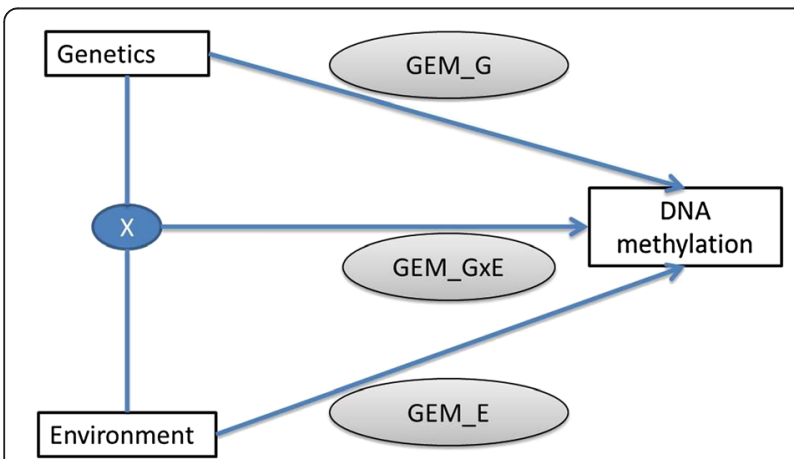

Fig. 1 A schematic of the analyses performed within Teh et al. [17]. Gmodel sequentially tests the association of 1423 methylation marks with 708,365 genotypes (and covariates), Emodel sequentially tests the association of 1423 methylation marks with 19 environmental factors and GxEmodel sequentially tests that association of 1423 methylation marks with the interaction of every combination of the 708,365 genotypes and 19 environmental factors. covariates cvrt can be simplified into the calculation of inner product of the projects of $\mathrm{x}$ and $\mathrm{y}$ as $\boldsymbol{r}_{\tilde{x} \tilde{y}}=\langle\tilde{\boldsymbol{x}}, \tilde{\boldsymbol{y}}\rangle$ and estimation of the test statistics.

Shabalin [23] also demonstrated the strategy to slice the large matrix into a small "blocks" in the correlation calculation for memory efficiency, which make the software able to handle data matrix with millions of rows and columns feasible in normal computational setting.

GEM tools called MatrixEQTL [23] library and implemented the below models which were used in [18],

$$
\mathrm{GEM}_{\text {Emodel }}: \boldsymbol{M}=\alpha+\beta \boldsymbol{E}+\gamma \boldsymbol{c \nu r t}+\varepsilon,
$$

which was implemented by calling matrixEQTL with "modelLINEAR", replacing gene expression with methylation, and SNP with environmental data.

$$
\mathrm{GEM}_{\mathrm{Gmodel}}: \boldsymbol{M}=\alpha+\beta \boldsymbol{G}+\gamma \boldsymbol{c v r t}+\boldsymbol{\varepsilon},
$$

which was implemented by calling matrixEQTL with "modelLINEAR", replacing gene expression with methylation.

\section{GEMGxEmodel : $\boldsymbol{M}$}

$$
=\alpha+\beta \boldsymbol{G} \times \boldsymbol{E}+\gamma \boldsymbol{c} v \boldsymbol{r t}+\boldsymbol{\varepsilon},
$$

which was implemented by calling matrixEQTL with "modelLINEAR_CROSS", replacing gene expression with methylation.

Emodel finds the association between methylation and environment genome-wide by performing millions of linear regression ( $\mathrm{N}=$ number_of_CpGs). The output of Emodel for particular phenotype, environmental factor or disease trait is a list of CpGs that are potential epigenetic biomarkers, as in Table 1.

Table 2 demonstrates the pseudo code that used $\mathbf{l m}$ function by iterative loops for Gmodel, we denoted it as LM_Gmodel. The best fit is chosen by the largest $R$ squared value.

Replacing the linear regression equation (line 6) in Table 2 by "fit $<-\operatorname{summary}(\operatorname{lm}(\mathrm{M}(\mathrm{i}) \sim \mathrm{G}(\mathrm{j}) * \mathrm{E}+$ cvrt))", produces the pseudo code for the implementation of LM_GxE model. The output of GxEmodel is a list of CpG-SNP-Env triplets, indicating the CpG-Env association segregated by genotype. The significant association of each triplet implies the methylation change is determined by the interplay between genotyping and environment. Both implementations indicate the number of linear regression as $\mathrm{N}=$ number_of_SNPs x number_of_CpGs. N could be billions of linear regressions engendering a very substantial computational task. However, using GEM tools, calculation of methQTLs and GxE interactions can be accomplished with much improved computational efficiency. 
Table 3 Benchmarking time consumption of GEM implementations on Emodel, Gmodel and GxEmodel by comparing normal R script in a public available dataset in standard laptop and HPC settings

\begin{tabular}{|c|c|c|c|c|}
\hline & Dataset: Teh et al., 1423 CpGs, & and 19 environmer & ard laptop & \\
\hline Method & Time cost on standard laptop & Time cost in HPC & Method & Time cost \\
\hline LM_Emodel & $95.1 \mathrm{~s}$ & & GEM_Emodel & $18.9 \mathrm{~s}$ \\
\hline LM_Gmodel & $>=60$ days $\left({ }^{a}\right)$ & $3 \mathrm{~h}$ & GEM_Gmodel & $5.2 \mathrm{~min}$ \\
\hline LM_GXEmodel & $>=60$ days $\left({ }^{a}\right)$ & $21 \mathrm{~h}$ & GEM_GxEmodel & $1.5 \mathrm{~h}$ \\
\hline
\end{tabular}

The time for LM_Gmodel and LM_GxEmodel in standard laptop was computed based on the time cost on 10 CpGs

\section{Results}

To benchmark GEM suite, we used the dataset from Teh et al. [18]. The standard laptop used for time comparisons had a $2.2 \mathrm{GHz}$ processor, $8 \mathrm{G}$ RAM, a windows 7 operating system and was 64 bit, which is typical in an academic setting. The HPC structure had eight parallel processes of each with eight core CPUs.

In [18], we studied the 1423 variably methylated regions from the methylomes of 237 neonates, and their association with 708,365 genetic variants and nineteen environmental factors made up of maternal conditions and birth outcomes. The methylome and genotype data are publically available at the NCBI Gene Expression Omnibus (GEO: http://www.ncbi.nlm.nih.gov/geo/ under accession numbers GSE53816 and GSE54445.) Environmental factors were simulated.

A schematic of the analyses performed is shown in Fig. 1. When the original analyses were conducted, multivariate regression models were applied sequentially in a HPC environment. For the same dataset, we compared the time taken to implement LM_Gmodel, LM_GxEmodel and LM_Emodel in a standard and HPC computational environment with

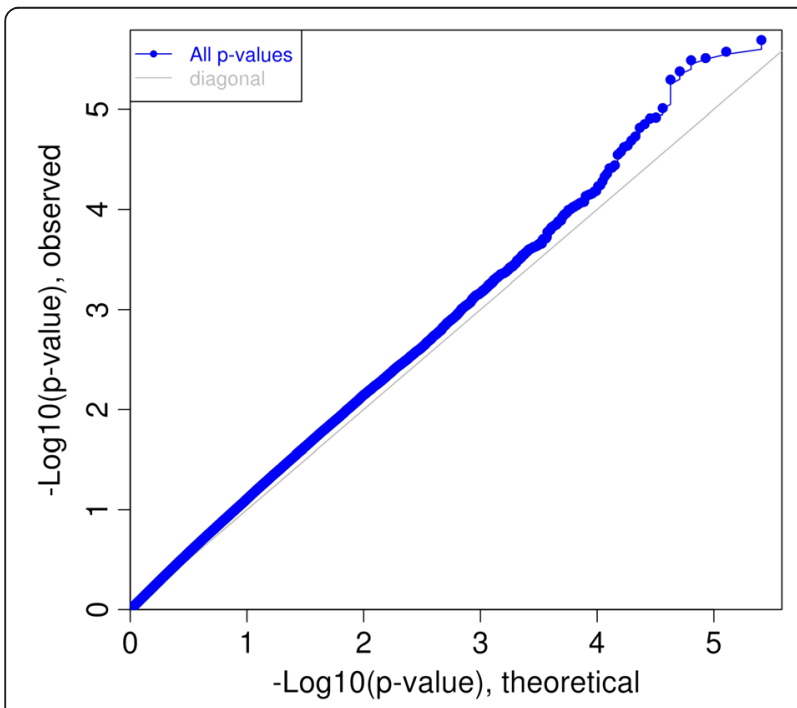

Fig. 2 QQplot for pvalues from iterations of 250,000 CpGs with an environment factor. It was produced by GEM_Emodel the time taken to implement GEM_Gmodel, GEM_ GxEmodel and GEM_Emodel on a standard laptop (Table 3).

\section{Benchmarking GEM_Emodel}

A substantial time saving was achieved using GEM_Emodel compared to standard sequential regression as LM_Emodel as in Table 1. (19 s compared to $95 \mathrm{~s}$ for 19 Emodels on 1423 CpGs). Results achieved were identical. In addition, GEM_Emodel has the option to create Q-Q plot for theoretical distribution and observed distribution on $\mathrm{p}$ values for every environment e.g. Fig. 2.

As subject numbers increase, computational time to run sequential models increases exponentially, whilst computational time in GEM_Emodel increases linearly. Figure 3 shows the computational time required for one Emodel on 100-1000 subjects for 250,000 CpGs.

\section{Benchmarking GEM_Gmodel}

In the original analysis [18], the regression equation (Eq. 1 and Table 1) used built-in $1 m$ function in $\mathrm{R}$ script, which we denoted as LM_Gmodel, was applied to each of the 1423 VMRs, cycling through the 708,365 SNPs, adjusted by sex as the covariate, resulting in 1008 million regression models. We compared the LM_Gmodel with

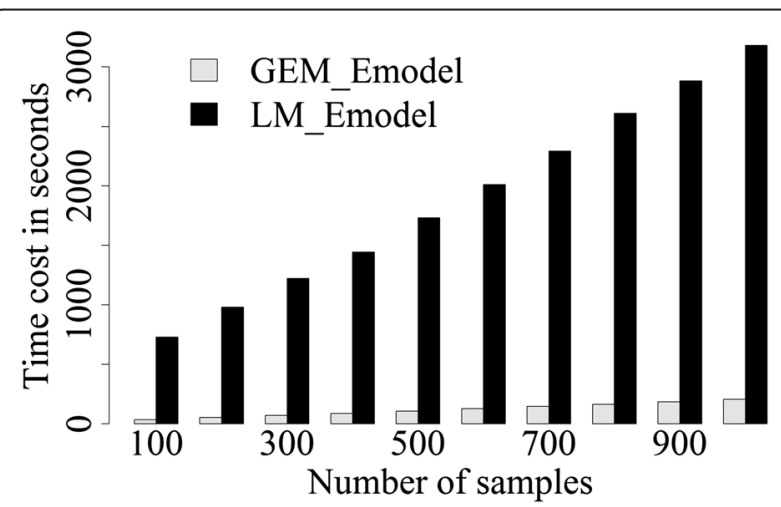

Fig. 3 The operation time consumption benchmark on the associations for 250,000 CpGs and one environmental factor and one covariate for the sequential number of samples from 100 to 1000. X-axis is the number of subjects, and $y$-axis is the time consumption in seconds. The benchmarking is done in a personal computer (processor $=2.2 \mathrm{GHz}, \mathrm{RAM}=8 \mathrm{G}$, system $=$ window7 64bit) 


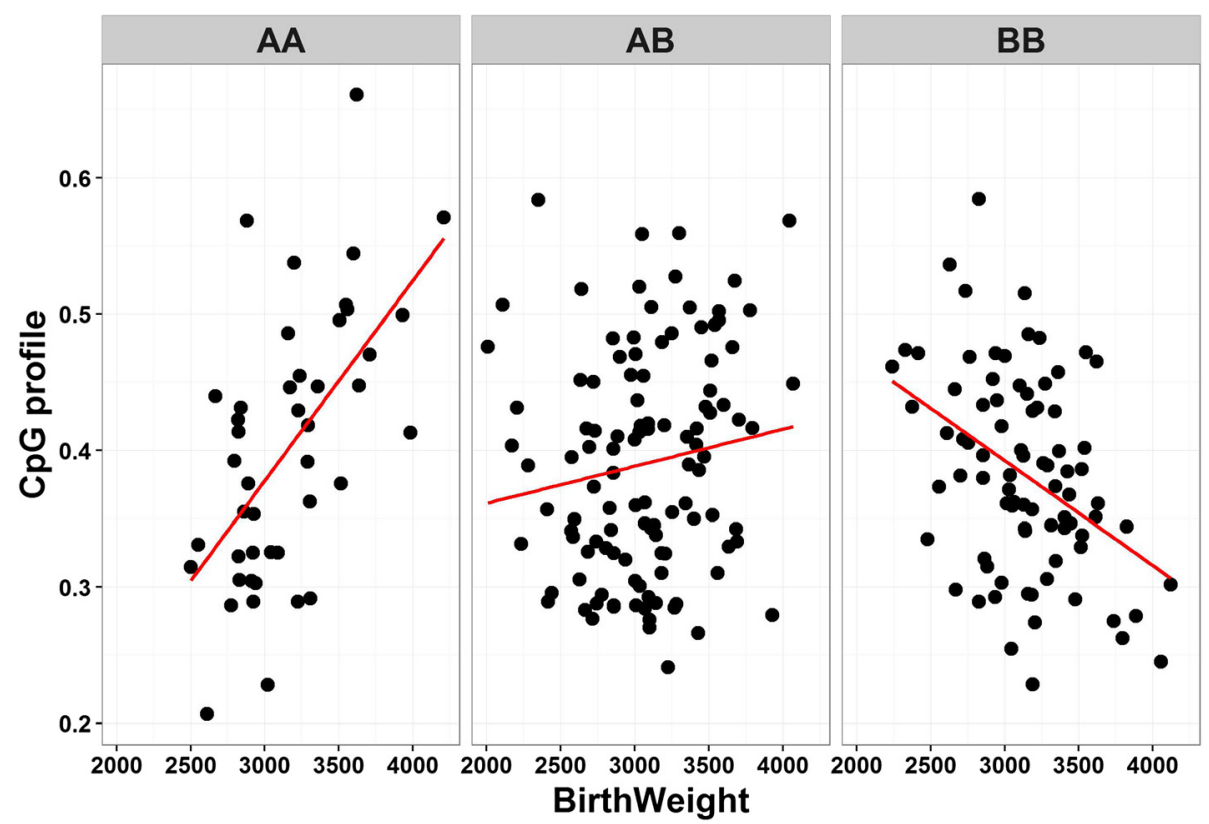

Fig. 4 The scatter plot to display an example of methylation corresponding to the environment in different genotype groups. $A A, A B$ and $B B$ are pseudo codes for major allele homozygote, heterozygote and minor allele homozygote. Phenotypic values are shown on the $x$-axis, and methylation value in percentage on the $y$-axis. The straight lines fit for associations in each group

GEM_Gmodel by the result and computational efficiency in a standard laptop (processor $=2.2 \mathrm{GHz}, \mathrm{RAM}=8 \mathrm{G}$, system = window7, 64bit). We also used a HPC structure with eight parallel processes of each with eight core CPUs (denoted as HPC) to benchmark LM_Gmodel as a reference. The computational time on HPC was $3 \mathrm{~h}$, in a standard computational environment, computational time was estimated to be 61 days. The same data was processed by the GEM Gmodel. It took 5.2 min to accomplish the task on a standard laptop. The results were identical to

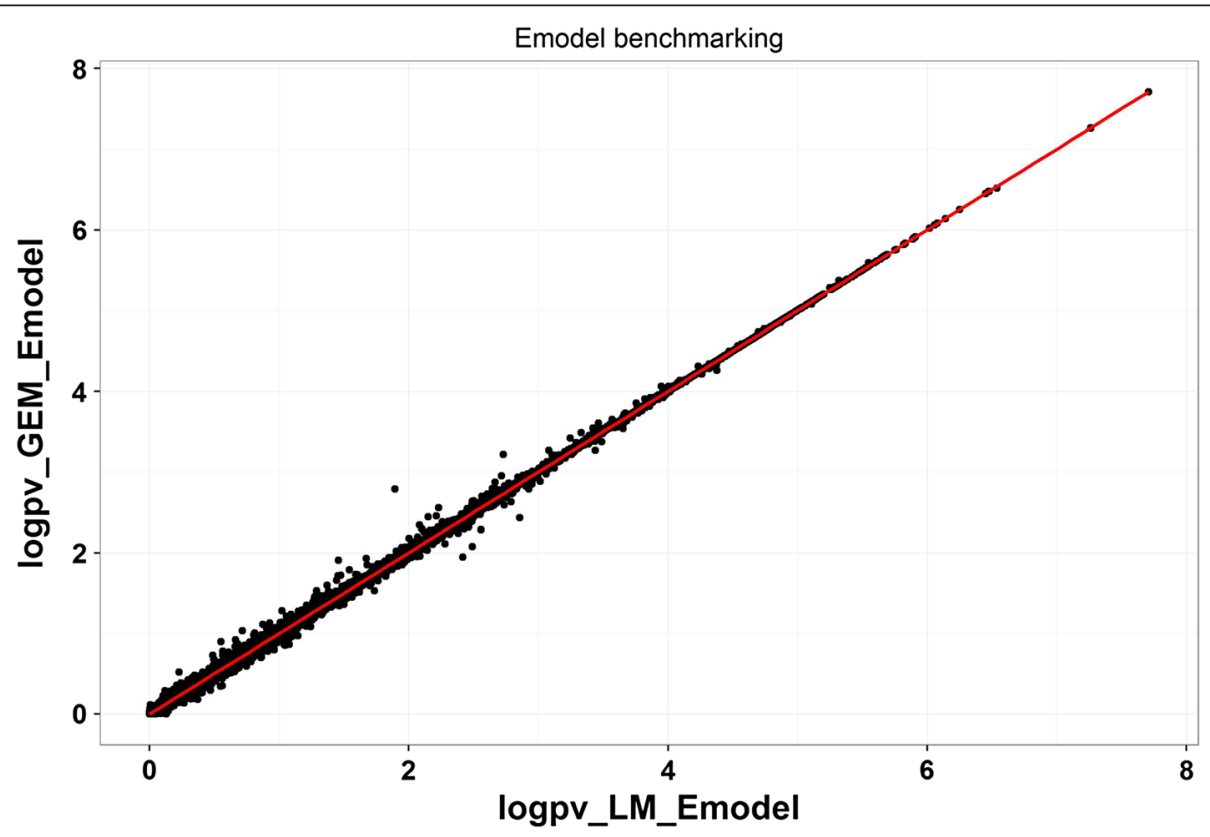

Fig. 5 Emodel benchmarking for methylation matrix containing missing values. Pvalue was transformed as -log10. A-axis is pvalues from LM_Emodel, $y$-axis is from GEM_Emodel. Among 250,000 CpGs that were tested, 18 \% of them contained at least one missing values. Our results showed pvalues for CpGs without missing values are perfectly matched, while there were slightly differences between the two implementations when CpG contains missing values 
those reported by Teh et al. [18] i.e. 12 disrupting pairs, 828 in cis- pairs and 583 in trans- pairs.

\section{Benchmarking GEM_GxEmodel}

The same scale of improvement in performance was achieved for the GEM_GxEmodel where each CpG was tested against the interaction of genotype at each of 708,365 SNPs with each of 19 environmental factors. This analysis originally took $21 \mathrm{~h}$ in the HPC environment and an estimated $>=60$ days on a standard laptop by using normal linear regression in R script, denoted as LM_GxEmodel. In the GEM_GxEmodel, it was accomplished in only $1.5 \mathrm{~h}$. The results were identical between analyses with identical $\mathrm{p}$-values for models containing all winning pairs of SNPs and environments (data not shown).

In addition GEM also has the option to produce a "segregation scatter plot" for methylation corresponding to environment in different genotype groups, for example, Fig. 4.

\section{Conclusion and discussion}

The advancements in genome-wide genotyping and DNA methylation assessment methods, coupled with wellcharacterized biological samples enable epigenetic association studies. GEM is designed for very fast testing of millions of hypotheses in epigenetics by using multiple linear regression models. It is suitable to the standard computing resources available to nearly all researchers.

GEM includes a graphic user interface for the convenience of researchers and does not require specialist computational knowledge, outside of the widely used R environment.

It should be noted that missing data requires careful handling in matrix-based operations. GEM uses the mean value to impute missing values if the data matrices supplied are incomplete. Figure 5 showed the p-values for GEM_Emodel and LM_Emodel are slightly different when the methylation matrix contains missing values. Researchers should assess the suitability of this imputation in the context of the individual study.

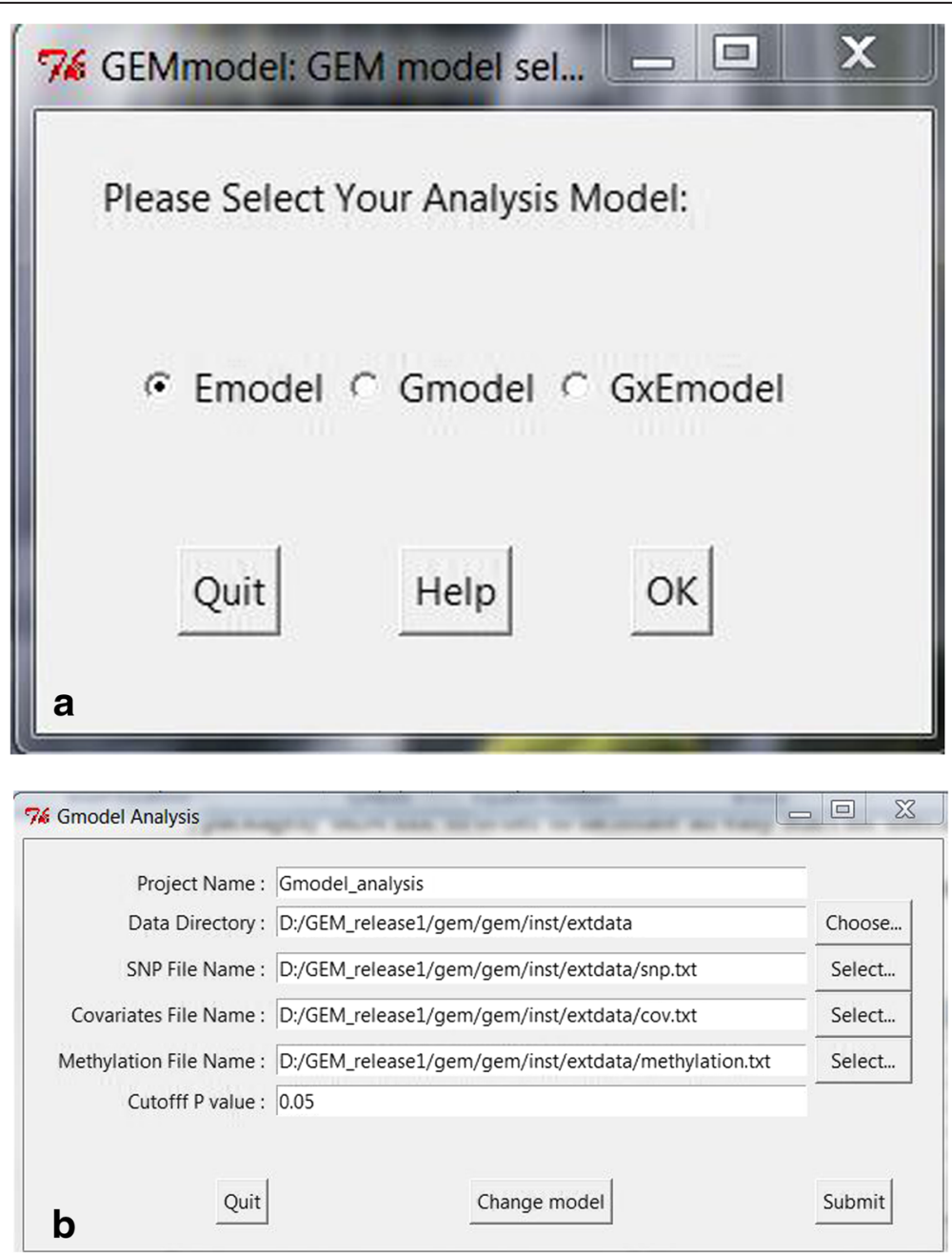

Fig. $\mathbf{6}$ GEM graphic user interface. $\mathbf{a}$ the selection of functions. $\mathbf{b}$ the file and parameter window 


\section{Abbreviations}

cis-, SNP and CpG locate in the same chromosome; EWAS, Epigenome wide association studies; GXE, the interaction of environmental and genetic influences; methQTLs, methylation quantitative trait loci; trans-, SNP and CpG locate in different chromosomes

\section{Acknowledgements}

Authors would like to thank Edmund Heng and Tran Nhat Sang for discussion and testing. Authors would also thank the A*STAR Computational Resource Centre through the use of its high performance computing facilities.

\section{Funding}

This research is supported by the Singapore National Research Foundation under its Translational and Clinical Research (TCR) Flagship Programme and administered by the Singapore Ministry of Health's National Medical Research Council (NMRC), Singapore- NMRC/TCR/004-NUS/2008 and NMRC/ TCR/012-NUHS/2014. Additional funding is provided by the Singapore Institute for Clinical Sciences, Agency for Science Technology and Research (A*STAR), Singapore and Singapore Ministry of Education Tier 2 grant, MOE2014T22023.

\section{Availability of data and materials}

GEM package can be downloaded from Bioconductor at https://www. bioconductor.org/packages/GEM/. In order to facilitate an easy start for users, we implement a graphic user interface for users to make the usage of the package, see Fig. 6

\section{Authors' contributions}

$\mathrm{HP}, \mathrm{JDH}$ and KN conceived of the study. HP wrote the GEM software and carried out the computational benchmarking. HP, JDH, KN and CKK wrote the manuscript. All authors read and approved the final manuscript.

\section{Competing interests}

The authors declare that they have no competing interests.

\section{Consent for publication}

Not applicable.

\section{Ethics approval and consent to participate}

Not applicable.

\section{Author details}

${ }^{1}$ Singapore Institute for Clinical Sciences (SICS), Agency for Science Technology and Research (A*STAR), Singapore 117609, Singapore. ${ }^{2}$ School of Computer Science and Engineering, Nanyang Technological University (NTU), Singapore 639798, Singapore. ${ }^{3}$ Yong Loo Lin School of Medicine, National University of Singapore (NUS), Singapore 119228, Singapore.

Received: 19 March 2016 Accepted: 21 July 2016

Published online: 02 August 2016

\section{References}

1. Holbrook JD. An epigenetic escape route. Trends in genetics : TIG. 2015; 31(1):2-4.

2. Murphy TM, Mill J. Epigenetics in health and disease: heralding the EWAS era. Lancet. 2014;383(9933):1952-4.

3. Ng JW, Barrett LM, Wong A, Kuh D, Smith GD, Relton CL. The role of longitudinal cohort studies in epigenetic epidemiology: challenges and opportunities. Genome Biol. 2012:13(6):246.

4. Bibikova M, Barnes B, Tsan C, Ho V, Klotzle B, Le JM, Delano D, Zhang L, Schroth GP, Gunderson KL, et al. High density DNA methylation array with single CpG site resolution. Genomics. 2011;98(4):288-95.

5. Meissner A, Gnirke A, Bell GW, Ramsahoye B, Lander ES, Jaenisch R. Reduced representation bisulfite sequencing for comparative high-resolution DNA methylation analysis. Nucleic Acids Res. 2005;33(18):5868-77.

6. Allum F, Shao X, Guenard F, Simon MM, Busche S, Caron M, Lambourne J, Lessard J, Tandre K, Hedman AK, et al. Characterization of functional methylomes by next-generation capture sequencing identifies nove disease-associated variants. Nat Commun. 2015;6:7211.

7. Teh AL, Pan H, Lin X, Lim YI, Patro CP, Cheong CY, Gong M, Maclsaac JL, Kwoh CK, Meaney MJ, Kobor MS, Chong YS, Gluckman PD, Holbrook JD,
Karnani N. Comparison of Methyl-capture Sequencing vs. Infinium 450K methylation array for methylome analysis in clinical samples. Epigenetics. 2016;11(1):36-48.

8. Rakyan VK, Down TA, Balding DJ, Beck S. Epigenome-wide association studies for common human diseases. Nat Rev Genet. 2011;12(8):529-41.

9. Lam LL, Emberly E, Fraser HB, Neumann SM, Chen E, Miller GE, Kobor MS. Factors underlying variable DNA methylation in a human community cohort. Proc Natl Acad Sci U S A. 2012;109(Suppl 2):17253-60.

10. Heijmans BT, Mill J. Commentary: The seven plagues of epigenetic epidemiology. Int J Epidemiol. 2012;41(1):74-8.

11. Houseman EA, Accomando WP, Koestler DC, Christensen BC, Marsit CI, Nelson $\mathrm{HH}$, Wiencke JK, Kelsey KT. DNA methylation arrays as surrogate measures of cell mixture distribution. BMC Bioinformatics. 2012;13:86.

12. Houseman EA, Molitor J, Marsit CJ. Reference-free cell mixture adjustments in analysis of DNA methylation data. Bioinformatics. 2014;30(10):1431-9.

13. Bell JT, Pai AA, Pickrell JK, Gaffney DJ, Pique-Regi R, Degner JF, Gilad Y, Pritchard JK. DNA methylation patterns associate with genetic and gene expression variation in HapMap cell lines. Genome Biol. 2011;12(1):R10.

14. Zhang D, Cheng L, Badner JA, Chen C, Chen Q, Luo W, Craig DW, Redman M, Gershon ES, Liu C. Genetic control of individual differences in gene-specific methylation in human brain. Am J Hum Genet. 2010;86(3):411-9.

15. Gibbs JR, van der Brug MP, Hernandez DG, Traynor BJ, Nalls MA, Lai SL, Arepalli S, Dillman A, Rafferty IP, Troncoso J, et al. Abundant quantitative trait loci exist for DNA methylation and gene expression in human brain. PLoS Genet. 2010;6(5):e1000952.

16. Sun YV. The Influences of Genetic and Environmental Factors on Methylome-wide Association Studies for Human Diseases. Current genetic medicine reports. 2014;2(4):261-70.

17. Lemire M, Zaidi SH, Ban M, Ge B, Aissi D, Germain M, Kassam I, Wang M, Zanke BW, Gagnon F, et al. Long-range epigenetic regulation is conferred by genetic variation located at thousands of independent loci. Nat Commun. 2015:6:6326.

18. Teh AL, Pan H, Chen L, Ong ML, Dogra S, Wong J, Maclsaac JL, Mah SM McEwen LM, Saw SM, et al. The effect of genotype and in utero environment on interindividual variation in neonate DNA methylomes. Genome Res. 2014:24(7):1064-74.

19. Liu Y, Aryee MJ, Padyukov L, Fallin MD, Hesselberg E, Runarsson A, Reinius L, Acevedo N, Taub M, Ronninger $\mathrm{M}$, et al. Epigenome-wide association data implicate DNA methylation as an intermediary of genetic risk in rheumatoid arthritis. Nat Biotechnol. 2013;31(2):142-7.

20. Chen L, Pan H, Tuan TA, Teh AL, Maclsaac JL, Mah SM, McEwen LM, Li Y, Chen $\mathrm{H}$, Broekman BF, et al. Brain-derived neurotrophic factor (BDNF) Val66Met polymorphism influences the association of the methylome with maternal anxiety and neonatal brain volumes. Dev Psychopathol. 2015;27(1):137-50.

21. Pan $H$, Lin X, Wu Y, Chen L, Teh AL, Soh SE, Lee YS, Tint MT, Maclsaac IL, Morin AM, Tan KH, Yap F, Saw SM, Kobor MS, Meaney MJ, Godfrey KM, Chong YS, Gluckman PD, Karnani N, Holbrook JD. GUSTO Study Group. HIF3A association with adiposity: the story begins before birth. Epigenomics. 2015;7(6):937-50

22. Ocana K, de Oliveira D. Parallel computing in genomic research: advances and applications. Advances and applications in bioinformatics and chemistry: AABC. 2015;8:23-35

23. Shabalin AA. Matrix eQTL: ultra fast eQTL analysis via large matrix operations. Bioinformatics. 2012;28(10):1353-8.

\section{Submit your next manuscript to BioMed Central} and we will help you at every step:

- We accept pre-submission inquiries

- Our selector tool helps you to find the most relevant journal

- We provide round the clock customer support

- Convenient online submission

- Thorough peer review

- Inclusion in PubMed and all major indexing services

- Maximum visibility for your research

Submit your manuscript at www biomedcentral com/submit
Biomed Central 